\title{
Hox Temporal Collinearity: Misleading Fallacy or Essential Developmental Mechanism?
}

\section{Abstract}

Kondo and collaborators recently reported the absence of Hox temporal collinearity in Xenopus tropicalis. They found none in the initiation of accumulation of Hox transcripts (detected via RNA seq). And none in the initial expression sequence of primary unprorocessed transcripts (Identified by using qRT-PCR against introns or intron-exon boundaries). Nor in the initial acquisition by Hox gene DNA of a mark for active chromatin. These findings are in conflict with the idea that temporal collinearity has to do with the initiation of Hox gene transcription or with the opening of and a progression from repressed to active states in Hox chromatin. But collinear acquisition of the same active chromatin mark has been shown by others in murine 5' Hoxd cluster genes. The reason for this difference is unknown. This careful study thus indicated that the initiation phase of Hox expression shows no temporal collinearity in X. tropicalis. A previous study in $X$. laevis from the same group also showed that the sequence of times for reaching (normalised) half maximal Hox expression showed no temporal collinearity. These conclusions are likely to be correct. These authors do however also conclude that "experimental evidence for the temporal collinearity hypothesis is not strong" There is however strong evidence that Hox temporal collinearity does occur in early vertebrate embryos. Below. I present and discuss 3 lines of evidence to resolve the present conflict I argue that Hox temporal collinearity actually does exist and that it is part of a central mechanism in early development.

\section{A.J.Durston, Institute of Biology, University of Leiden}

Keywords: hox genes; temporal collinearity; axial patterning; gastrulation; xenopus

\section{1/ Introduction}

Kondo and collaborators ${ }^{1}$ recently reported the absence of Hox temporal collinearity in Xenopus tropicalis. They found none in the initiation of accumulation of Hox transcripts (detected via RNA seq). And none in the initial expression sequence of primary unprorocessed transcripts 
(Identified by using qRT-PCR against introns or intron-exon boundaries). Nor in the initial acquisition by Hox gene DNA of a mark for active chromatin ${ }^{1}$. These findings are in conflict with the idea that temporal collinearity has to do with the initiation of Hox gene transcription or with the opening of and a progression from repressed to active states in Hox chromatin. But collinear acquisition of the same active chromatin mark has been shown by others in murine $5^{\prime}$ Hoxd cluster genes ${ }^{2}$. The reason for this difference is unknown. This careful study thus indicated that the initiation phase of Hox expression shows no temporal collinearity in X. tropicalis. A previous study in $X$. laevis from the same group ${ }^{3}$ also showed that the sequence of times for reaching (normalised) half maximal Hox expression showed no temporal collinearity ${ }^{3}$. These conclusions are likely to be correct. These authors do however also conclude that "experimental evidence for the temporal collinearity hypothesis is not strong" There is however strong evidence that Hox temporal collinearity does occur in early vertebrate embryos. Below. I present and discuss 3 lines of evidence to resolve the present conflict I argue that Hox temporal collinearity actually does exist and that it is part of a central mechanism in early development.

\section{2/ Temporal Collinearity in the Literature}

Several groups have presented impressive evidence that Hox temporal collinearity actually does exist in different early vertebrate embryos, including Xenopus ${ }^{4-15}$ For discussion, see ${ }^{16}$ Particularly important is that some studies used in situ hybridisation as the method of monitoring $H o x$ expression. This enabled specific examination of Hox expression in tissues that show temporal collinearity, while excluding background from those that do not. These studies therefore give the clearest data. For details, see s-8, 10,11. $^{6}$.

\section{3/ An Evaluation of Temporal Collinearity in $X$. Tropicalis: Direct Comparison with the Study by Kondo et al.}

Investigation using the same principal data source as Kondo et $\mathrm{al}^{1}$ (a publicly available RNA seq. database ${ }^{17}$ ) shows that there is substantial Hox temporal collinearity in $X$. tropicalis. This tendency towards temporal collinearity is seen during the first major increase in Hox expression, leading to the first main Hox expression peak, between Stages 10.5 (10h) and St.25 (25h). The Hox transcript numbers at these stages are potentially enough to mediate a developmental function ${ }^{18}$. In the $X$. tropicalis Hoxd cluster (most temporally collinear), 8 out of the 8 Hox 
genes expressed, are potentially all in a clear temporally collinear sequence for most of this early trajectory. Two (Hoxd8, Hoxd9) are out of sequence for some of the time and in sequence for the rest due to having unusually early initiation times combined with a prolonged period of low level expression before they take their place in the temporal collinearity sequence and two more (Hoxd4, Hoxd9) are ambiguous. They are each expressed close to expression of a neighbouring Hox gene (Hoxd3 and Hoxd10, respectively). Sometimes one gene is ahead. Sometimes the other. The other $X$. tropicalis Hox clusters show temporal collinearity as well as exceptions. In the Hoxa cluster, Hoxa1, Hoxa2. Hoxa3, Hoxa4, Hoxa5, Hoxa11, Hoxa13 show potential temporal collinearity. Hoxa9, Hoxa10, Hoxa6, Hoxa7 are out of sequence and Hoxxa4, Hoxa5 are ambiguous. In the Hoxc cluster, Hoxc6, Hoxc9, Hoxc10, Hoxc11, hoxc12, show potential temporal collinearity; Hoxc4, Hoxc5, Hoxc8 Hoxc13 are out of sequence In the Hoxb cluster Hoxb1, Hoxb3, Hoxb4, Hoxb8, Hoxb9 are potentially in a collinear temporal sequence. , are out of sequence. Hoxb4, Hoxb8 are ambiguous. In total, 25 out of the 38 Hox genes examined show potential temporal collinearity and 13 do not. It is clear that most but possibly not all Hox genes probably show temporal collinearity in this phase of development. There is thus clearly at least a subclass of Hox genes that show early temporal collinearity.

This tendency for temporal collinearity above is thus apparently not absolute. There are exceptions. These may relate to the fact that RNA seq. is not the ideal method for recording Hox expression. Previous studies using a suitable method (in situ hybridisation) show $100 \%$ temporal collinearity. It is also unsurprising that Hox initiation was not temporally collinear. Xenopus Hox genes show diverse behaviour in initiation. Some are maternally expressed. Others are not. Some have initially low expression that rises later. Others show swiftly rising expression. See below (Hoxd cluster, Fig. 1b,) It is also unsurprprising that normalised Hox expression maxima showed no temporal collinearity or regularity ${ }^{3}$. Different Hox genes have very different expression profiles, no doubt associated with having different functions at various developmental stages ${ }^{19,20}$. In contrast with Kondo et al's apparent assumption, I feel it is very unlikely that Hox chromatin opening and initiation of Hox transcription are confined to the initiation phase of general Hox expression. Successive Hox functions in different tissues will determine that these activities are required and occur at many 
different times in development. In fact it is already known that Hox chromatin opens at least twice during development: in early development and during the initial phase of limb development ${ }^{9}$.

\section{4/ The Developmental Importance of Hox Temporal Collinearity}

Nieuwkoop and collaborators first showed that the Amphibian A-P axis is made in a timed manner. First the forebrain is induced, then progressively more posterior parts all the way back to the tail ${ }^{21,22}$. These findings showed that the axial neural tissue is first specified as anterior (presumptive forebrain: telencephlon/diencephalon) and then sequentially posteriorised. This transformation involved first a conversion to presumptive mesencephalon, and subsequently to presumptive rhombencephalon, and then to presumptive spinal cord. These findings were confirmed by more recent studies in various vertebrates $7,23,24,25,26$.

There is evidence that timed A-P axis formation in Xenopus is mediated by time space translation (TST) from gastrula stages onwards ${ }^{7,27}$ (Fig. 2). Hox temporal collinearity acts as a BMP dependent timer () in the non-organiser mesoderm (NOM) of the early embryo. Another embryonic region, Spemann's organiser (SO) emits anti-BMP signals. Timed application of either an intact organiser or the organiser anti-BMP signal noggin to an organniserless embryo at sequential stages blocked the timer at sequential A-P Hox values, and thereby fixed timed cell states sequentially, leading to Hox spatial collinearity. Either the treatment generated an anteriorly truncated axis with the truncation at sequentially more posterior positions for sequentially later treatments (implanted organisers; continuous anti-BMP sources) or it generated one or more A-P/ Hox zones; sequentially later treatments gjving sequentially more posterior zones (noggin) ${ }^{7}$. In the absence of an organiser signal or noggin, temporal collinearity proceeded as normal, in NOM but no spatially collinear Hox pattern was ever generated. Hox expression then died out. We conclude that sequentially repeated interactions between the two embryonic parts lead to small populations of cells being fixed successively at sequential space-time points/Hox codes. Presumably, specific events including morphogenetic cell movements in the embryo or possibly Hox timed mesodermal cell ingression ${ }^{8}$, cause these sequentially timed/zoned populations to be arranged in and specify an anterior early to posterior late spatial Hox sequence that becomes the initial A-P pattern.. This mechanism was first revealed in Xenopus but there is evidence that it operates during gastrulation and later stages in other vertebrates. The conversion of a dynamically changing temporally collinear Hox sequence to a stable spatially collinear axial Hox pattern 
of A-P positional information by timed anti- BMP also operates in early chicken and zebrafish embryos ${ }^{28-30}$. It has been shown that $H o x$ temporal collinearity in the chicken gastrula determines the order in which primitive streak cells migrate to the node ${ }^{8,11}$, that a population of dynamically changing primitive streak cells interacts with the a stable organiser derived cell population to generate the early axial pattern in mouse embryos ${ }^{31}$ (, and that Hox temporal collinearity during chicken gastrulation generates positional information (e.g., forelimb position) in later development $\left.{ }^{44}\right)$. These parallel and complementary findings in other vertebrate embryos establish that this Hox timing mechanism is conserved in evolution. Interestingly, the discoveries above define a believable role for the Spemann organiser, which is well known to be important in A-P patterning. I note that organiser-less (ventralised) Xenopus embryos show temporal but not spatial Hox collinearity and that reimplantation of an organiser reintroduces and fixes the spatial pattern exactly as predicted above ${ }^{7}$.

During these stages, function of the Hox genes themselves is clearly a part of the timing and time space translation mechanisms. The timing mechanism operates and generating the spatial Hox pattern involves autoregulation and collinear cross-regulation among the Hox genes and Hox associated miRNA's, leading to a sequence of collinear interactions among them. This is thus at least partly a Hox cascade mechanism. The fact that Hox cascades are involved in specifying the axis is clearly demonstrated by the cascade of phenotypes generated by Hox gain and loss of function experiments in Xenopus and other systems ${ }^{32-39}$. We have called this aspect of collinearity: macrocollinearity ${ }^{40}$.

\section{Hox Temporal Collinearity in a Cell Line}

In an important early paper that received too little attention ${ }^{32}$ Faiella et al. demonstrated that NT2/D1 cells show Hox temporal collinearity (though they do not make a spatial Hox pattern). NT2/D1 was not just any old cell line: these are human pluripotent embryonal carcinoma (EC) cells. They have properties in common with the types of cells that display temporal collinearity in embryos (NOM mesoderm and primitive streak). The fact that these E.C. cells can not generate stable Hox expression or a Hox pattern presumably reflects the absence of any organiser function in this cell line. This study was the first to reveal the Hox-Hox interaction that underlies temporal collinearity (posterior induction (PI)). Interestingly, the cascade LOF phenotypes generated in temporal collinearity in this cell line are mimicked by some of the Hox LOF phenotypes in spatial collinearity generated in the Xenopus embryo ${ }^{33-36}$. 
This is as expected and is a further endorsement of the proven connection between temporal and spatial collinearity.

\section{Conclusion}

In conclusion, while accepting the new findings by Kondo et al., I doubt their contention that "experimental evidence for the temporal collinearity hypothesis is not strong". Hox temporal collinearity appears to occur in a population of pluripotent stem cells in the early vertebrate embryo, and is part of the embryo-building program.

\section{Acknowledgement}

I thank M. Owens, Mike Gilchrist, Mustafa Khokha and their coauthors (Owens et al., 2017) and the Crick Institute and their IT department for allowing us to use their excellent searchable high time resolution database of Xenopus tropicalis developmental transcripts. Without this fantastic database, this investigation would never have been undertaken. Figure 1B is taken directly from it. I am also very grateful to Mike Gilchrist and Mustafa Khokha for helpful comments and especially for their very generous and important help and advice regarding using the database. Mustafa and Mike: thank you both very much indeed!!

\section{References}

1 Kondo M. ${ }^{*}$, Matsuo M. 2,5, Igarashi K. 2,6, Yoshikazu Haramoto3, Takayoshi Yamamoto2,7, Yuuri Yasuoka4,8, Masanori Taira2hde novo transcription of multiple Hox cluster genes takes place simultaneously in early Xenopus tropicalis embryos Company of Biologists ,9ttp://bio.biologists.org/ by guest on March 14, 2019 2 Soshnikova N, and Duboule D. Epigenetic Temporal Control of Mouse Hox Genes in Vivo. Science 2009; 324: 1320-1323.

3 Kondo M., Yamamoto T, Takahashi S. et al. Comprehensive analyses of hox gene expression in Xenopus laevis embryos and adult tissues. Develop. Growth Differ. 2017; 59: 526-539

4 Moreau C. Paolo Caldarelli1,2,3, Didier Rocancourt1,2, Julian Roussel1 ,2,4, Nicolas Denans5, Olivier Pourquie6 and Jerome Gros1,2 (2019)Timed collinear activation of Hox genes during gastrulation controls the avian forelimb position Current Biology, DOI: https://doi.org/10.1016/j.cub.2018.11.009

5 Izpisúa-Belmonte J C, Falkenstein H, Dollé P, et al.. Murine genes related to the Drosophila AbdB homeotic genes are sequentially 
expressed during development of the posterior part of the body. EMBO J. 1991; 10(8): 2279-2289.

6 Gaunt S, and Strachan L. Temporal colinearity in expression of anterior Hox genes in developing chick embryos Developmental Dynamics 1996; 207(3):270-80.

7 Wacker S, Jansen, $\mathrm{H}$, McNulty $\mathrm{C}$, et al. Timed interactions between the Hox expressing non-organiser mesoderm and the Spemann organiser generate positional information during vertebrate gastrulation. Dev Biol. 2004;268(1):207-19.

8 limuraT. \& Pourquie O. Collinear activation of Hoxb genes during gastrulation is linked to mesoderm cell ingression. Nature. 2006; 442: 568-571.

9 Soshnikova N, and Duboule D. Epigenetic Temporal Control of Mouse Hox Genes in Vivo. Science 2009; 324: 1320-1323.

10 Gouveia A, Marcelino HM, Gonçalves L, Et al. Patterning in time and space: HoxB cluster gene expression in the developing chick embryo, Cell Cycle. 2015; 14(1): 135-145, DOI: 10.4161/15384101.2014.972868 11 Denans N., limura T, Pourquie O. Hox genes control vertebrate body elongation by collinear Wnt repression. eLife 2015;4:e04379. DOI:

10.7554/eLife.04379

12 Pascual-Anaya J, Sato I, Sugahara F, et al. Hagfish and lamprey Hox genes reveal conservation of temporal colinearity in vertebrates. Nat Ecol Evol.2018;2(5):859-866.

13 Deschamps J. and Duboule D. (2018) Embryonic timing, axial stem cells, chromatin dynamics, and the Hox clock Genes and Development. 2018; 31:1406-1416.

14 Monteiro AS, and Ferrier D.E.K. Hox genes are not always Colinear Int J Biol Sci. 2006; 2(3): 95-103. .

15 Duboule D. The rise and fall of Hox gene clusters. Development. 2007; 134: 2549-2560.

16 Durston A. J. (20199) Vertebrate hox temporal collinearity: does it exist and what is it's function?, Cell Cycle, 18:5, 523-530, DOI: 10.1080/15384101.2019.1577652

17 Owens ND, Blitz IL, Lane MA, Patrushev I, Overton JD, Gilchrist MJ, Cho KW, Khokha MK. (2016) Measuring Absolute RNA Copy Numbers at High Temporal Resolution Reveals Transcriptome Kinetics in Development. Cell Rep. 2016 Jan 6. pii: S2211-1247(15)01491-6. 18 Pare A, Kosman D, ${ }^{1}$ Beaver W, ${ }^{2}$ Freund $Y,{ }^{2}$ and McGinnis W.(2009) Transcriptional analysis of the Hox gene Scr at single molecule resolution yields evidence for transcriptional bursting during Drosophila embryogenesis, Curr Biol. 19(23): 2037-2042. 19 Weatherbee SD, Halder G, Kim J, Hudson A, Carroll S.(1998) Ultrabithorax regulates genes at several levels of the wing- 
patterning hierarchy to shape the development of

the Drosophila haltere Genes \& Dev. 12: 1474-1482

20 Pavlopoulos A, and Akam M. (2011) Hox

gene Ultrabithorax regulates distinct sets of target genes at successive stages of Drosophila haltere morphogenesis. Proc Natl Acad Sci U S A. 2011 Feb 15; 108(7): 2855-2860.

21 Nieuwkoop PD. (1952) Activation and organisation of the central nervous system in Amphibians. III. Synthesis of a working hypothesis. J. Exp. Zool. 1952;120: 83-108.

22 Eyal Giladi H. Dynamic aspects of neural induction in Amphibia. In: Amphibians. Vaillant-Carmanne H; 1954. 81pp.

23 Gamse J, Sive H (2000) Vertebrate anteroposterior patterning: the Xenopus neurectoderm as a paradigm. BioEssays 22:976-986. doi: 0.1002/1521-1878(200011)22:11<976::AID-BIES4>3.0.CO;2-C 24 Gamse JT, Sive H (2001) Early anteroposterior division of the presumptive neurectoderm in Xenopus. Mech Dev 104:21-36. doi: 0.1016/S0925-4773(01)00358-

25 Vasiliauskas D, and Stern CD, (2001) Patterning the Embryonic Axis: FGF Signaling and How Vertebrate Embryos Measure Time Cell, Vol. 106, 133-136, July 27, 2001.

26 Stern CD, Charité J, Deschamps J, et al (2006) Head-tail patterning of the vertebrate embryo: One, two or many unresolved problems? Int J Dev Biol 50:3-15. doi: 10.1387/ijdb.052095cs.

27 Durston AJ, Zhu K. A time space translation hypothesis for vertebrate axial patterning. Seminars in Cell \& Developmental Biology. 2015;42:86-93.

28 Tucker JA, Mintzer KA, Mullins MC. (2008)The BMP signaling gradient patterns dorsoventral tissues in a temporally progressive manner along the anteroposterior axis. Dev Cell. 2008;14(1):108-19 29 Hashiguchi M, Mullins MC. ( 2014) Anteroposterior and dorsoventral patterning are coordinated by an identical patterning clock.

Development. 140(9):1970-8

30 Dias, A. S., de Almeida, I., Belmonte, J. M., Glazier, J. A., and Stern, C. D. (2014). Somites without a clock. Science 43, 791-795. doi:

$10.1126 /$ science. 12

47575

31 Wymeersch FJ., Stavroula Skylaki3, Yali Huang1, Julia A. Watson1, Constantinos Economou1, Carylyn Marek-Johnston1, Simon R. Tomlinson1, Valerie Wilson ${ }^{*}$ (2018) Transcriptionally dynamic progenitor populations organised around a stable niche drive axial patterning Development. 17 December 2018 as 10.1242/dev.168161 http://dev.biologists.org/lookup/doi/10.1242/dev.168161 
32 Faiella A, Zappavigna V. 36 Mavilio F, and Boncinelli E. Inhibition of retinoic acid-induced activation of 3 ' human $\mathrm{HOXB}$ genes by antisense oligonucleotides affects sequential activation of genes located upstream in the four HOX clusters. Proc. Nadl. Acad. Sci. USA Vol. 91, pp. 53355339, June 1994

33 McNulty C, Peres J, van den Akker W, Bardine N, Durston A. Knockdown of the complete Hox paralogous group 1 leads to dramatic hindbrain and neural crest defects. Development 2005;132(June (12)):2861-71

34 Zhu K, Spaink HP, Durston AJ, (2017) Hoxc6 Loss of Function Truncates the Main Body Axis in Xenopus. Cell cycle, In Press. 35 Zhu, K., Spaink HP, Antony J. Durston (2017c) Collinear Hox- Hox interactions are involved in patterning the vertebrate anteroposterior $(A-P)$ axis PLoS ONE in press.

36 Hooiveld, M. Morgan, R., In der Rieden, P Houtzager, E., Pannese, M., Damen, K., Boncinelli, E., and Durston, A. (1999) Novel colinear interactions between vertebrate Hox genes. Int. J. Dev. Biol. 43:665-74. 37 Yekta S. ${ }^{*} \neq$, Tabin CJ, and Bartel DP. (2008) MicroRNAs in the Hox network: an apparent link to posterior prevalence. Nature reviews genetics 9, 189-796

38 Woltering J. M., and Durston A, (2008) MiR10 represses HoxB1a and HoxB3a in Zebrafish. PLoS ONE. 2008 Jan 2;3(1):e1396.

39 Bardine N, Lamers G, Wacker S, Donow C, Knoechel W, Durston A. (2014) Vertical signalling involves transmission of Hox information from gastrula mesoderm to neurectoderm. PLoS ONE 2014;9(12):e115208, http://dx.doi.org/10.1371/journal.pone.0115208 47575

40 Durston AJ, (2018) Two Tier Hox Collinearity Mediates Vertebrate Axial Patterning. Frontiers in Cell and Developmental Biology, Perspective doi: 10.3389/fcell.2018.00102

\section{Figure Legends}

\section{Fig. 1 Temporal collinearity and deviations from it in $\mathbf{X}$. tropicalis}

1A Temporal order $r$ of Hox expression in the D,A,C,B clusters. Hox genes that re out oif the temporally collinear sequence are marked in red.

1B Hoxd cluster: detailed expression profiles. Note that Hoxd1, Hoxd8 and Hoxd9 start their expression much too early. Their temporally 
collinear positions are regained at a slightly later stage due to a prolonged period of low level jnitial expression.

\section{Fig. 2. Timing, Axial patterning, and Time Space Translation}

Above: The domain structure of the vertebrate A-P axis Domains with significant Hox genes and other markers. An unexpected element is introduced by the newly characterised EAD: extreme anterior domain, which makes the face. This is shown as the most anterior part of the straight axis. Actually. the anterior end of the dorsal A-P axis bends around to the ventral side of the embryo to face posteriorly- like the handle of a walking stick (not shown). A Head: anterior head (corresponding to telencephalon, diencephalon, mesencephalon). $\mathrm{P}$ Head: posterior head (corresponding to anterior rhombencephalon, occipital somites). Neck: cervical somites, posterior rhombencephalon, Thorax: thoracic vertebrae, anterior spinal cord. Abdomen: Lumbar and sacral regions, spinal cord. Tail: caudal vertebrae, spinal cord.

Above and below: Time space translation. A biological timer, represented by the clockface below, proceeds from 1-12 (red numbers). The timer starts with information needed for making the EAD, proceeds to the anterior head, then to posterior head, then to neck, then to thororax, then abdomen, then tail. The timer needs $B M P$ to function so is in tissues like NOM with high BMP (yellow/orange ). Anti-BMP (blue) (produced by the organiser) interacts with the timer sequentially to freeze the identities of an early/A-late/P sequence of axial zones. In the axial sequence, th e Hox genes are each both a component of the timer at their appropriate times and are sequentially involved in setting up the A-P sequence of axial zones. The genes involved in time space translation in the EAD-head zones are unknown. The heavy red dashed arrows represent transport of cells from the high BMP environment (yellow) to the BMP inhibited environment (blue). The continuous dark red arrows connecting yellow to blue for the head and EAD I indicate that the details he re are unknown. The head and tail of the A-P timer are close together because of their representation on a clockface. No statement about molecular identities is intended.

Fig. 3. Hox sequences for axial cascade phenotypes_Above: Wild type Hox sequence. second and third down: blue diagrams: Loss of function affecting temporal collinearity in ES cells. Hox 1 and Hox3 LOF each cut off the Hox temporal collinearity sequence from their paralogue 
position. Fourth and fifth down: Xenopus spatial collinearity, loss of function. Fourth: Hox 1 loss of function (LOF) (all 3 Hox 1 genes knocked down by morpholinos (MOs)). The axis from Hox 1 backward is compromised. The dotted line indicates there is still reduced residual expression for some posterior Hox genes. Fifth down:Hoxc6 LOF (MO) The axis from Hoxc6 backward is compromised/deleted. Sixth down: Hoxb4 gain of function (GOF): ectopic expression of Hoxb4 in Hox free dorsalised embryoss. A partial posterior axis is generated, starting with Hoxb4. Seventh down: Hoxb9 gain of function. Details similarly as above for Hoxb4 gain of function. Please note that: In Hox 1 loss of function, the immediately more anterior marker (Gbx2) has enhanced expression, presumably reflecting absence of posterior dominance exerted by Hox 1 genes. Similarly, in Hoxc6 loss of function, Hox4 and Hox5 are hyperinduced, presumably reflecting absence of Hoxc6 posterior dominance.

\section{Figures}

Fig. 1

A

\begin{tabular}{|l|l|l|l|l|l|l|l|l|l|l|l|l|}
\hline Cluster & & & & Para & Expr & Seq & & & & & & \\
\hline Hoxd & d1 & d3 & d4 & d8 & d9 & d10 & d11 & d13 & & & & \\
\hline Hoxa & a1 & a2 & a7 & a9 & a3 & a10 & a5 & a4 & & a6 & a11 & a13 \\
\hline Hoxc & c6 & c4 & c9 & c8 & c5 & c10 & c3 & c11 & c12=c13 & & & \\
\hline Hoxb & b1 & b3 & b4 & b8 & b9 & b7 & b6 & b2 & b5 & & & \\
\hline
\end{tabular}


B

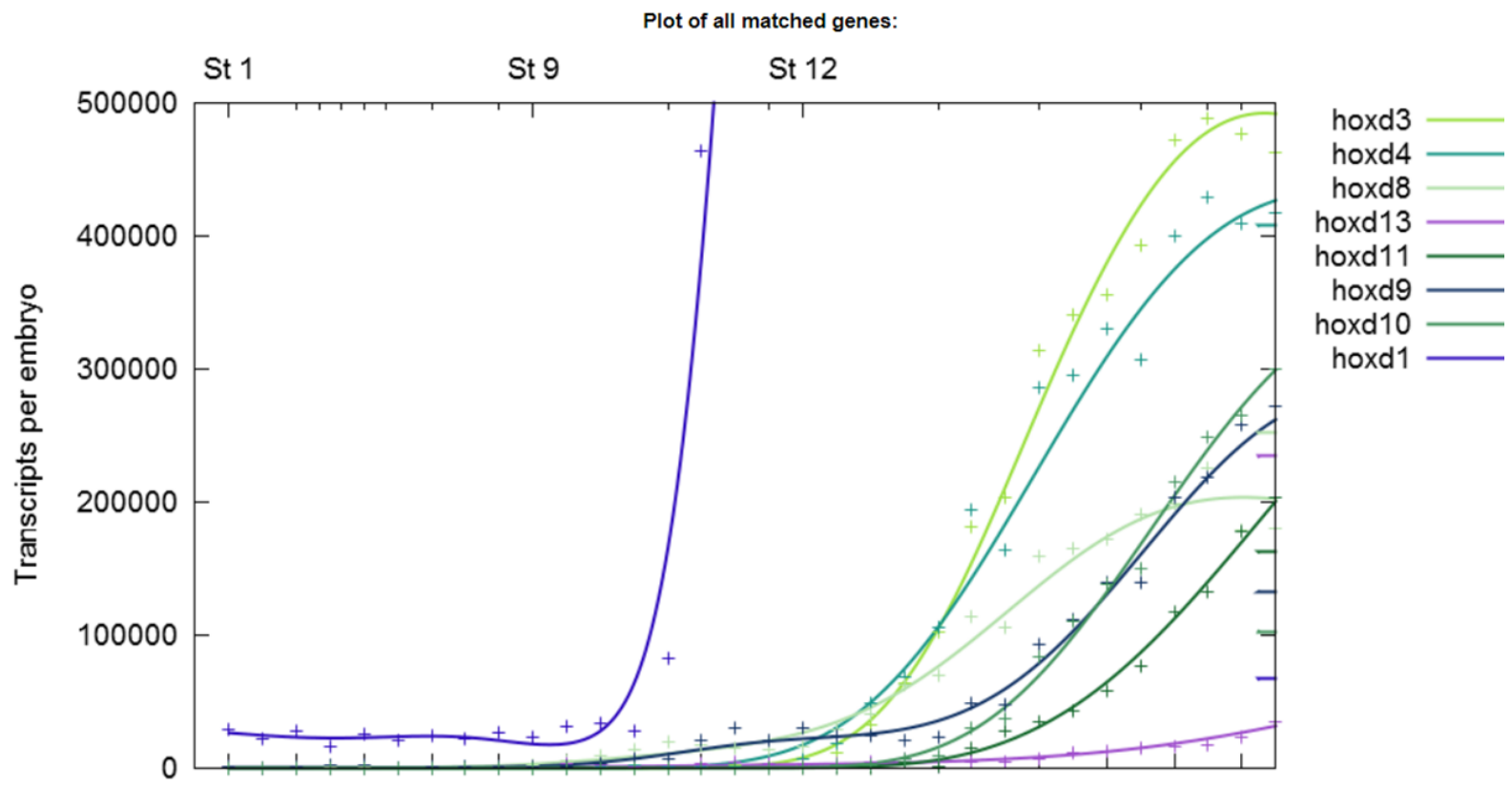

Fig. 2 


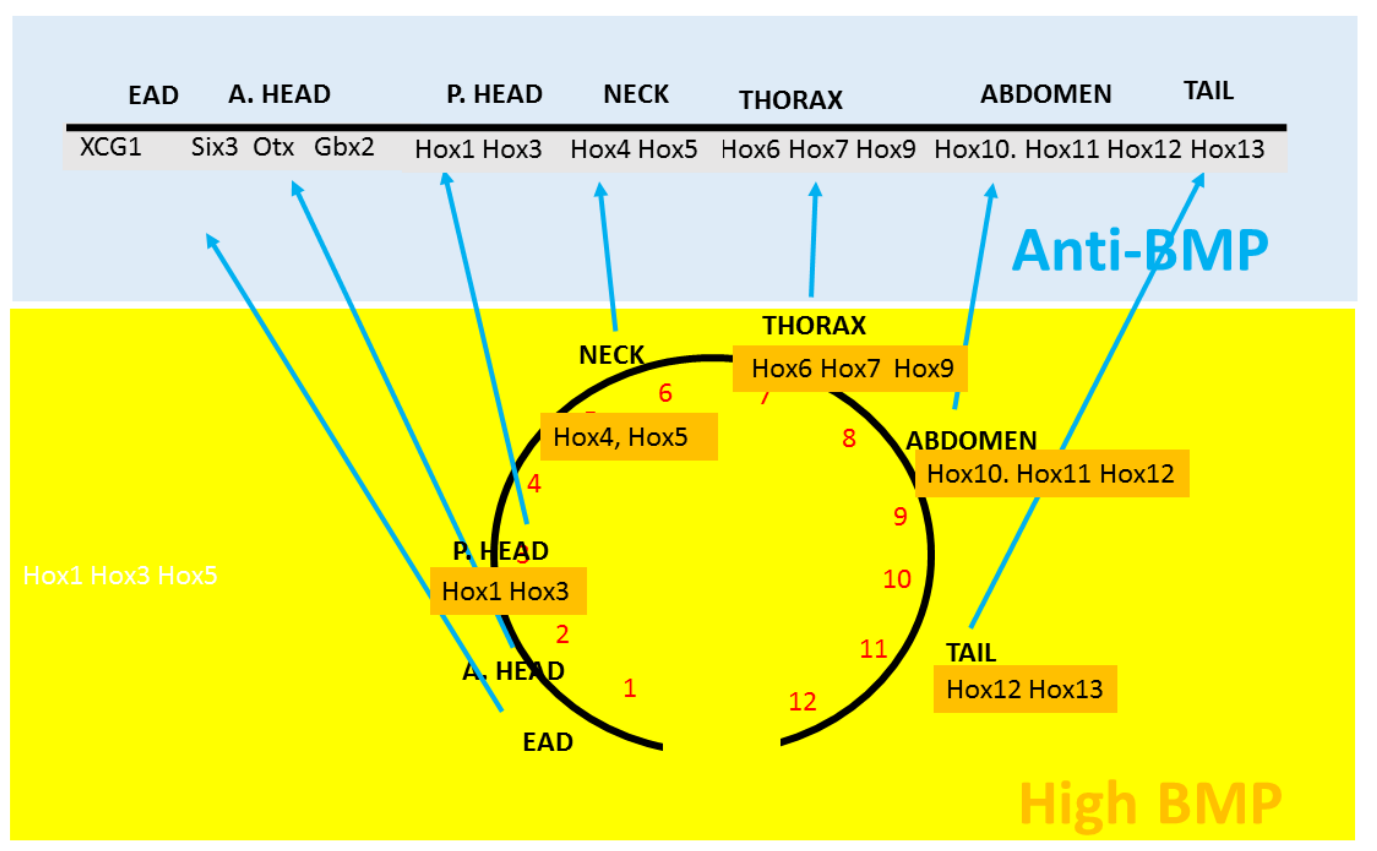

Fig. 3

Wt.

XAG1 Six3 Otx2 Gbx2 Hox1 Hox2 Hox3 Hox4 Hox5 Hox6 Hox7 Hox8 Hox9 Hox10 Hox11 Hox12 Hox13

T Hoxb1-

T Hoxb3-

S Hox1-

S Hoxc6-
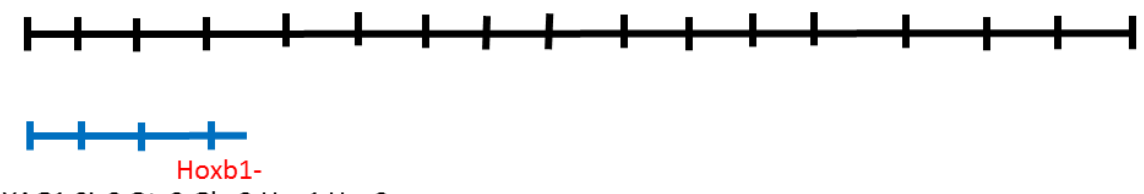

XAG1 Six3 Otx2 Gbx2 Hox1 Hox2

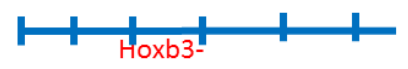

XAG1 Six3 Otx2 Gbx2 r

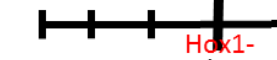

XAG1 Six3 Otx2 Gbx2 Hox1 Hox2 Hox3 Hor4 Hax5

XAG1 Six3 Otx2 Gbx2 Hox1 Hox2 Hox3 Hox4 Hox5 Hox6 Hox7 Hox8 Hox9 Hox10 Hox11 Hox12 Hox13

S Hoxb4+

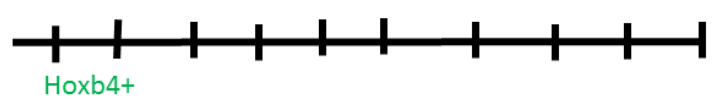

Hox9 Hox10 Hox11 Hox12 Hox13

S Hoxb9+

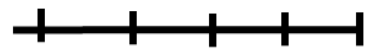

Hoxb9+ 